Política y Sociedad

ISSN: $\quad 1130-8001$

ISSN-e: $1988-3129$

\title{
Medios de comunicación y esfera pública: el caso del tratamiento por la prensa española de las políticas públicas en torno a la ley de extranjería
}

\author{
Antonio Prieto-Andrés ${ }^{1}$ y Cayetano Fernández Romero ${ }^{2}$
}

Recibido: 30-12-2018 / Aceptado: 02-12-2019

Resumen. El objetivo de este trabajo es analizar, desde una metodología de análisis de contenido mixto, cuantitativo y cualitativo, cómo la prensa contribuye a la conformación de la esfera pública, a partir del estudio del caso del tratamiento de las políticas públicas en torno a la ley de extranjería española. Para lograrlo, se va a poner especial atención a la selección que hacen los seis diarios analizados de sus fuentes, las categorías temáticas que se presentan y los discursos predominantes, más o menos proclives al fenómeno migratorio, que prevalecen en ellos. Resultado de este estudio de caso, que abarca el periodo 2003-2016, se observará una preferencia mayoritaria de las fuentes oficiales sobre las de la sociedad civil, y una clara relación entre la tipología de las fuentes preferidas por cada uno de los seis medios analizados con las temáticas que se presentan y el tipo de discurso sobre la inmigración predominante. En definitiva, la prensa va a ejercer un papel de mera correa de transmisión de los mensajes oficiales, con lo que se pone en tela de juicio el rol mediador que aquella debería tener en una sociedad democrática, contribuyendo así decisivamente a la conformación de una esfera pública plural.

Palabras clave: esfera pública; inmigración; ley de extranjería; prensa; fuentes de información; discurso.

\section{[en] Mass Media and the Public Sphere: The Case of the Spanish Press's Treatment of the Public Policies Surrounding the Foreigners Act}

\begin{abstract}
The aim of this work is to analyze, with a mixed, quantitative and qualitative content analysis methodology, how written press contributes to create the public sphere regarding migration, by means of a case study about the treatment of migration policies related to the Spanish Foreigners Act. For this purpose, we will examine the selection made by six newspapers of their sources, topic categories and dominant discourses in favor of or against the migration phenomenon. With the results obtained in the case study, which covers the period 2003-2016, we can infer a clear preference for official sources over social ones and a clear relation between favourite source types in each one of the six newspapers analysed, with their topics and dominant discourses about immigration. As a conclusion, the press is acting merely as a transmission belt for the official message, and thus makes us doubtt the mediating role that it should have in a democratic society, by contributing in a determining way in the formation of a plural public sphere.
\end{abstract}

Keywords: public sphere; immigration; Foreigners Act; press; information sources; discourse.

\footnotetext{
Universidad San Jorge, Zaragoza (España).

E-mail: aprieto@usj.es

2 Universidad San Jorge, Zaragoza (España).

E-mail: cfernandez@usj.es
} 
Cómo citar: Prieto-Andrés, A. y C. Fernández Romero (2020): “Medios de comunicación y esfera pública: el caso del tratamiento por la prensa española de las políticas públicas en torno a la ley de extranjería", Política y Sociedad, 57(1), pp. 121-141.

Sumario. 1. Introducción y estado de la cuestión. 2. Fuentes y metodología. 3. Resultados. 4. Conclusiones. 5. Bibliografía.

Agradecimientos. Este trabajo ha sido financiado gracias a la colaboración del Programa Operativo FEDER Aragón 2014-2020, “Construyendo Europa desde Aragón” (Grupo de Investigación S05_17D).

\section{Introducción y estado de la cuestión}

La inmigración es uno de los fenómenos más relevantes de la agenda internacional del presente siglo. También en España se trata de un asunto de especial interés, ya que nuestro país ha pasado, en menos de veinticinco años, de ser un país de emigración a receptor de inmigrantes, por lo que es un asunto fundamental en la esfera pública y mediática. Aunque la prensa ha tratado sistemáticamente las diferentes vertientes de la inmigración en España, como la demográfica, la socioeconómica o la cultural, entre otras, en este trabajo nos vamos a centrar en la jurídico-política. La Ley Orgánica 4/2000, de 11 de enero, sobre derechos y libertades de los extranjeros en España y su integración social, conocida popularmente como "ley de extranjería", es la norma que regula esta cuestión en España. En este ámbito, la prensa se ha hecho eco de temas como los derechos y deberes de los extranjeros, cómo se ha de proceder a su regularización en caso de encontrarse de forma ilegal, cómo controlar mejor las fronteras, o cuáles son las políticas de las administraciones públicas (a nivel estatal, autonómico y local, a las que denominaremos, simplemente, políticas públicas) en este ámbito, aspecto que va a ser objeto de análisis en este trabajo.

Este trabajo se va a centrar en mostrar cómo la prensa hace sus aportaciones a la esfera pública cuando habla de las políticas públicas sobre la gestión de la inmigración, a partir de sus referencias a la ley de extranjería. Para lograr este objetivo, se van a tomar como punto de referencia tres aspectos: a) qué categorías temáticas se muestran; b) qué fuentes principales emplea la prensa para documentarse y c) qué tipo de discursos se manifiestan, así como la relación existente entre todos estos elementos.

Pero antes es oportuno conceptualizar el término "esfera pública", así como el papel que juegan los medios de comunicación en dicho ámbito. Para Habermas (1974: 124) se trataría de un campo de nuestra vida social donde "los ciudadanos se relacionan voluntariamente bajo la garantía de que pueden unirse para expresar y publicar libremente opiniones, que tengan que ver con asuntos relativos al interés general. En el marco de una gran concurrencia, esta comunicación necesita de determinados medios de transmisión y de influencia; tales medios de la esfera de lo público son hoy: periódicos y revistas, radio y televisión. Hablamos de la esfera política de lo público". A estos habría que añadir hoy las redes sociales en Internet. Se trataría de una esfera mediadora entre sociedad y Estado, en la que se forma la opinión pública, y que permite un control democrático de la acción estatal.

La esfera pública funciona legitimando democráticamente la acción del Estado al seleccionar los asuntos que son relevantes para la toma de decisiones políticas y 
agregándolos en las opiniones públicas que compiten entre sí por la preponderancia, a través de argumentos más o menos bien informados. Así, la comunicación pública sería una fuerza que estimula y orienta las opiniones y deseos de los ciudadanos, mientras, a la vez, compele al sistema político a adaptarse y ser más transparente (Habermas, 2009: 135).

El periódico debe ser mediador y creador de una discusión pública fortalecida, y no solo un simple órgano de transmisión de noticias. Cuando la prensa se convierte en medio de masas, en empresa que busca el lucro y cuyo contenido está pensado para el consumo, para Habermas ha perdido una parte importante de su función mediadora dentro de la esfera pública, puesto que preponderarían otros intereses de carácter económico que la desvirtuarían. Esta postura ha sido muy criticada por autores como Thompson (1996), para quien es muy dudoso que la recepción de los productos de los medios de comunicación de masas pueda ser considerada como un acto manipulado e irreflexivo de consumo, por lo que se estaría exagerando la pasividad de los individuos. Además, no podemos olvidar que el desarrollo de los medios de comunicación (como la prensa digital) ha creado una nueva clase de comunicación pública que permite la interacción de los lectores en tiempo real, introduciendo un nuevo elemento en este planteamiento (Pecourt, 2015).

Gomis (1991) distingue entre la tarea propia de los medios, que es la interpretación; y su función política, que es la mediación. Como mediador, su función es aproximar a autoridades y sociedad civil, proponiendo modos de integrar los intereses de ambos. El rol de mediador del periódico será más perfecto cuanto menor sea la diferencia entre la cantidad de hechos provocados por el sistema político y el provocado por el ambiente social. Si el periódico se limita a trasladar al ambiente social las salidas (outputs) o productos del sistema político, ejercerá el papel de transmisor, pero no de mediador. Si es al contrario, entonces ejerce de mediador. En esta misma línea, Casero y López (2012) también consideran que la mediación es una de las funciones normativas del periodismo en democracia. Borrat (1989) cree que el análisis del discurso periodístico permite rastrear en los textos indicios relevantes de las decisiones tomadas por el periódico en cuanto a excluir, incluir y jerarquizar a los actores y las fuentes de la información política, cuyo análisis permitirá precisar cuál es la línea política del periódico y cuáles los objetivos que moldean sus prácticas.

Además, Habermas considera de vital importancia el mantenimiento de la prensa de calidad que, en el ámbito de la comunicación política, en la que los lectores actúan como ciudadanos, juega el papel de "medio líder" (Habermas, 2009: 134), por encima de la radio y la televisión, gracias a la capacidad de aquella de generar reportajes y análisis extensos y razonados. Sin una información de calidad creada a partir de una investigación profunda y experta, con diversidad de fuentes, la comunicación política perdería su vitalidad discursiva. La prensa influye en ese sistema político a través de las opiniones publicadas a partir de diferentes fuentes y actores (políticos y partidos, lobistas, grupos de presión o actores de la sociedad civil), que son seleccionados por los profesionales de los medios de comunicación para sus receptores, a los que influye en mayor o menor medida (Habermas, 2006).

Así pues, la fuente informativa es el inicio de todo proceso comunicativo, ya que sin aquella no existiría el mensaje (Pérez Curiel, 2005). Manuel López define 
la fuente informativa como "un canal — persona o institución- que nos proporciona datos sustanciales o complementarios -al ser testigo directo o indirecto- para poder confeccionar noticias, reportajes, crónicas e informes" (López, 1995:31). Si se abunda en ello, hay que recordar que las fuentes tienen sus propios intereses a la hora de decidir promover el conocimiento de determinados datos y argumentos, y no otros, por lo que la tarea de filtrado por parte del periodista es imprescindible, para evitar convertirse en un mero portavoz de la fuente. Sobre este punto, Berganza y Chaparro (2012) coinciden con Ospina Hurtado (2004:1) al fijarse en la influencia que tiene la fuente sobre la determinación de los contenidos de los medios (la denominada agenda): "Es un término que designa a los individuos, los grupos, los centros de poder y las instituciones que, como actores de la sociedad, tienen la capacidad de comunicar algo y de influir en la agenda de los asuntos públicos que trata la información. Son las instituciones, grupos sociales o sujetos individuales y/o colectivos que proporcionan información sobre los acontecimientos; en últimas, son las instancias de poder, interesadas en validar y legitimar su visión de los hechos". De Fontcuberta (1993) considera que las diferencias entre los medios se basan en dos factores: la selección de fuentes informativas y la elección de los aspectos formales de la información. Borrat (1989) afirma que la potencia informativa del periódico se pondrá de manifiesto en el número, la calidad y el pluralismo de sus fuentes de información:

(...) cuanto mayor sea la cantidad, la calidad y diversidad de las informaciones que comunica y de las fuentes que cita, tanto mayor será su credibilidad y, por tanto, su influencia (...). Ante un hecho noticiable determinado, el periódico necesita disponer de varias fuentes contrastables para que su propia versión no sea una mera transcripción o reproducción de la que le ha proporcionado una sola fuente, ni refleje tan solo la versión de una de las partes del conflicto (Borrat, 1989: 54).

Las fuentes que emplean los medios van a determinar los intereses sociales que subyacen bajo una determinada política, como las políticas públicas en torno a la ley de extranjería en este trabajo, y juegan un papel destacado en la toma de posiciones sociales y políticas. Así, el estudio de las fuentes puede ayudar a comprobar si el periodismo es capaz de transmitir una información proporcionada y equitativa en toda la complejidad del hecho, o si solo recoge lo que promueven las clases dirigentes, especialmente en el ámbito político (Rodríguez y Bezunartea, 2015).

El segundo elemento analítico de este artículo se fija en los discursos sobre las políticas públicas en torno a la ley de extranjería presentes en las unidades de análisis. En este trabajo se siguen de forma muy especial las investigaciones de Zapata-Barrero $(2008,2009)$ sobre los aspectos discursivos relativos a la inmigración. También es relevante el trabajo de Casero (2007), que se fija en la identidad mediática de los inmigrantes, y que requiere una aproximación interdisciplinar, como la que se propone en esta investigación. Para ello se detiene en la función de los medios como auténticos gestores de identidades públicas, ya que, a través de sus discursos, aquellos determinan quiénes son representados y qué marcas y significados se asocian a cada colectivo. Además, observa la eficacia del 
uso de mecanismos discursivos para legitimar las políticas migratorias restrictivas, en línea con los trabajos de Martín y Whittaker (1998), y Wodak y Meyer (2003). Precisamente estos últimos autores, junto con De la Fuente (2007), Delgado (2007), Van Dijk (2007, 2008, 2009) y Van Dijk y Zapata-Barrero (2007) representan el enfoque de los que, desde los métodos del análisis crítico del discurso, se han centrado en el estudio del discurso racista y en la relación entre autóctonos y extranjeros. Finalmente, las investigaciones más centradas en los discursos parlamentarios sobre inmigración de Bañón y Fornieles (2008), Márquez (2007), Zapata-Barrero (2008, 2009) y Van Dijk y Zapata-Barrero (2007) han permitido comparar contenidos y categorías temáticas entre los discursos parlamentarios, mucho más extensos y elaborados, y los de la prensa, aspecto este que también se analiza en este trabajo, a partir del modelo metodológico que se explica en el siguiente epígrafe.

A partir de este planteamiento teórico, las preguntas que se responderán en este trabajo serán: a) ¿Qué dicen los medios cuando tratan el tema objeto de estudio?; b) ¿Cuáles son las fuentes principales que emplean los medios analizados y qué peso tienen las fuentes oficiales frente a las de la sociedad civil?; c) ¿Qué tipología discursiva aparece en la prensa analizada? y d) ¿Existe alguna relación entre las fuentes y los tipos de discursos?

\section{Fuentes y metodología}

Las unidades de análisis de esta investigación se han obtenido de seis diarios en su versión impresa: El País, El Mundo, ABC, Heraldo de Aragón, El Periódico de Aragón y La Vanguardia. Se seleccionaron todas aquellas que incluyeran conjuntamente los términos "ley" y "extranjería" entre el 1 de enero de 2003 y el 31 de diciembre de 2016. Se eligieron estos medios porque incluyen tres diarios nacionales de referencia, con líneas editoriales diferenciadas, así como otros tres diarios de ámbito regional, dos aragoneses y uno catalán. La inclusión de estos últimos pretende incorporar al estudio sensibilidades y enfoques distintos a los que aportan los diarios nacionales. Se encontraron 3.428 piezas en total. Para hacer viable la investigación se decidió reducir el número de unidades de análisis que compondrían el corpus, para lo que se practicó una técnica estadística denominada "muestreo aleatorio estratificado", que es "aquel en el que los elementos de la población se dividen en clases o estratos. La muestra se toma asignando primero, mediante algún criterio, un número de miembros a cada estrato y escogiendo después los elementos que forman la muestra por muestreo aleatorio simple dentro del estrato" (Peña y Romo, 1997: 272). En este caso, los estratos son los años en los que se han publicado las piezas de cada diario. Tras la realización de esta operación estadística, el corpus definitivo quedó circunscrito a 793 unidades de análisis. A partir de esta muestra, se codificaron diferentes bloques temáticos presentes en ellas, para poder realizar un análisis de contenido mixto, cuantitativo y cualitativo. Este trabajo se centra solo en el bloque de las políticas públicas relacionadas con la ley de extranjería, es decir, en todas aquellas unidades de análisis que, tras la codificación llevada a cabo, tratan el tema de cómo las administraciones públicas (estatales, autonómicas y locales) gestionan la 
inmigración con los instrumentos que aporta la ley de extranjería. En concreto, el reparto de unidades de análisis entre los seis diarios, tras el muestreo, es el siguiente (la primera cifra corresponde al número total de piezas; la segunda, entre paréntesis, es la resultante tras el muestreo, y la tercera, las unidades que tratan el tema de las políticas públicas): El País, 757 (122/33); El Mundo, 976 (157/42); ABC, 860 (139/39); Heraldo de Aragón, 171 (171/51); El Periódico de Aragón, $122(122 / 33)$ y La Vanguardia, 505 (82/23). El número de unidades que componen el corpus definitivo es de 221.

Respecto a la metodología, para este trabajo se ha optado por el análisis de contenido mixto, cuantitativo y cualitativo, que gira en torno a las herramientas empleadas para obtener los datos descriptivos de una serie de variables. Para Berelson (1952:18), el análisis de contenido se trata de una herramienta "para la descripción objetiva, sistemática y cuantitativa" de los textos comunicativos. Este método ofrece unos datos precisos a través de indicadores de frecuencia y de las interrelaciones existentes entre las variables evaluadas. El enfoque del análisis de contenido cualitativo (Krippendorff, 1990) está en el significado latente, que no es inmediatamente obvio, mientras que el análisis de contenido cuantitativo se fija en el significado manifiesto, literal. Para detectar ese significado latente, hay que fijarse en el contexto y no solo en la frase o párrafo, lo que ayuda a describir el material objeto de estudio.

Para analizar el papel de las fuentes informativas en cada uno de los diarios, en la investigación que da origen a este trabajo se incluyó un conjunto de variables que determinaba cuáles eran las fuentes principales y secundarias empleadas en cada unidad de análisis (diferentes administraciones públicas, gobiernos centrales, autonómicos o locales, partidos políticos de la oposición, ONG, abogados o sindicatos, entre otros, hasta un total de 23 posibles fuentes). Para este estudio se entiende por fuente principal aquella que se muestra claramente como preponderante en la pieza analizada. Esta preponderancia se valora atendiendo a los siguientes criterios: la mayor extensión de las manifestaciones de la fuente dentro del texto de la pieza en relación con las demás, así como la presencia de la mención a la fuente en el título, subtítulo, antetítulo y/o entradilla de la pieza.

En lo que interesa a esta investigación, se consideran fuentes oficiales las que representen a alguno de los poderes del Estado (ejecutivo, legislativo —en sus vertientes estatal, autonómica o local- y judicial), incluidos los partidos políticos, por su vinculación e influencia con los poderes ejecutivo y legislativo, para diferenciarlas de las fuentes institucionales, donde, además de las anteriores, se podría añadir a sindicatos, asociaciones de empresarios, ONG o cualquier otro tipo de entidad legalmente constituida. Las fuentes de la sociedad civil las compondría este segundo grupo de fuentes institucionales no oficiales, junto con los ciudadanos, bien de forma particular, bien agrupados en asociaciones, así como los profesionales y expertos de todos los ámbitos. Un tercer grupo lo formarían los propios medios de comunicación a través de sus editoriales, columnistas y colaboradores.

Para analizar los discursos, en esta investigación se sigue el planteamiento de Zapata-Barrero (2009), que estudia los discursos políticos sobre la inmigración a partir del conflicto entre monoculturalidad y multiculturalidad. En este marco, se pueden producir dos tipos de discursos (Zapata-Barrero, 2009:58): a) el que lleva al 
pasado monocultural, al que se denomina "reactivo" porque reacciona contra el proceso histórico y busca restablecer dicho pasado; b) el que lleva al futuro multicultural, denominado "proactivo", que asume la irreversibilidad del proceso y busca conformarlo como marco para orientar los cambios sociales. El discurso reactivo pretende gestionar el conflicto y se preocupa por las alteraciones negativas que supone el proceso de multiculturalidad. Lo interpreta como algo negativo, una amenaza. Es un conflicto de intereses entre ciudadanos e inmigrantes. Tiene en cuenta solo a los ciudadanos, que siempre tienen prioridad sobre los extranjeros. El discurso reactivo puede seguir dos tipos de retóricas: el ciudadanismo y el tradicionalismo. Por contra, el discurso proactivo aspira a proporcionar a las personas recursos para que gestionen los conflictos, ante su irreversibilidad histórica. Se ve como una oportunidad, un reto. El marco de referencia es toda la población, sea ciudadana o no, sea votante o no. Los discursos proactivos tienen la función de socializar el proceso para que todas las formas de expresión cultural puedan ser objeto de los principios de la democracia, siempre que todas las partes respeten el pluralismo. Las dos expresiones retóricas de este discurso son la retórica de la igualdad y la de la ciudadanía. En la Tabla 1 se muestran, esquemáticamente, cuáles son las ideas principales que sustentan cada una de las retóricas que conforman los discursos reactivos y proactivos, respectivamente.

Tabla 1. Ideas principales de las retóricas de los discursos reactivos y proactivos

\begin{tabular}{|c|c|}
\hline Discurso reactivo & Discurso proactivo \\
\hline $\begin{array}{l}\text { Retórica del ciudadanismo } \\
\square \quad \text { Populismo: prima el interés del } \\
\text { ciudadano frente al del inmigrante } \\
\text { en las zonas de conflicto } \\
\text { multiculturales. } \\
\square \quad \text { Esencialismo: los intereses y las } \\
\text { necesidades de los ciudadanos son } \\
\text { vistos como inmutables, como los } \\
\text { únicos criterios para construir un } \\
\text { discurso. }\end{array}$ & $\begin{array}{l}\text { Retórica de la ciudadanía } \\
\square \quad \text { Inclusión: el inmigrante forma parte de } \\
\text { un proceso que debe finalizar } \\
\text { necesariamente en el reconocimiento } \\
\text { pleno de la ciudadanía. } \\
\square \quad \text { Acomodación: la categoría del } \\
\text { inmigrante debe ir acompañada de la } \\
\text { del ciudadano, buscando argumentos } \\
\text { para su adaptación mutua. }\end{array}$ \\
\hline $\begin{array}{l}\text { Retórica del tradicionalismo } \\
\square \quad \text { Tradición e identidad nacional: } \\
\text { recurre al pasado para formar sus } \\
\text { argumentos. La inmigración } \\
\text { conlleva un conflicto que es } \\
\text { expresión de una alteración de los } \\
\text { valores de la tradición que sustentan } \\
\text { la estructura de la sociedad. } \\
\text { Defensor de la identidad nacional. } \\
\text { Concepto de autoridad: con el } \\
\text { proceso de multiculturalidad se } \\
\text { ponen en duda las estructuras de } \\
\text { autoridad que sustentan la sociedad } \\
\text { y las instituciones tradicionales, lo } \\
\text { que no es deseable. }\end{array}$ & $\begin{array}{l}\text { Retórica de la igualdad } \\
\square \quad \text { Carácter normativo: expresa la } \\
\text { necesidad de tener como criterio el } \\
\text { principio de igualdad de oportunidades } \\
\text { para gestionar las zonas de conflicto } \\
\text { multiculturales. No significa igualdad } \\
\text { como idéntico, sino como diferencia. } \\
\square \quad \text { Imparcialidad: no se privilegia una } \\
\text { cultura determinada al gestionar la } \\
\text { diferencia cultural. La idea básica es } \\
\text { que la diferencia cultural no produzca } \\
\text { o consolide desventajas por razones } \\
\text { que no dependan de la voluntad de la } \\
\text { persona. }\end{array}$ \\
\hline
\end{tabular}

Fuente: elaboración propia a partir de Zapata-Barrero (2009). 
A partir de este modelo, hemos categorizado, mediante las herramientas que ofrece el análisis de contenido, dónde se encuadran discursivamente las diferentes unidades de análisis, poniendo en relación la presencia de cada una de las cuatro retóricas posibles, dos pertenecientes al discurso reactivo (ciudadanismo y tradicionalismo) y dos al discurso proactivo (ciudadanía e igualdad), tanto si aparecen de forma única (si solo se encuentra un tipo de retórica en la unidad) o determinando si se produce la combinación entre dos de ellas (por ejemplo, tradicionalismo con igualdad) en una misma unidad de análisis. La forma de operacionalizar la identificación de las retóricas presentes en cada unidad ha sido analizar cada texto y determinar la presencia o no de las retóricas presentes en él, codificándolas según el planteamiento explicado. En total, han sido once las variables previstas, como se puede apreciar en la Tabla 4.

\section{Resultados}

Este apartado se estructura en tres bloques en torno al caso de estudio (las políticas públicas en relación con la ley de extranjería), que son: las categorías temáticas, las fuentes principales empleadas y los discursos pro y reactivos presentes en los seis medios analizados.

\subsection{Categorías temáticas vinculadas a las políticas públicas a partir de la ley de extranjería}

Una vez sometidas a escrutinio las unidades de análisis, se han detectado tres categorías temáticas: 1) el papel de la ley de extranjería como instrumento para gestionar los flujos migratorios; 2) la ley de extranjería en las comunidades autónomas (CC. AA.) y 3) la ley de extranjería en los ayuntamientos. Todas ellas presentan, a su vez, diversas subcategorías temáticas.

\subsubsection{El papel de la ley de extranjería como instrumento para gestionar los flujos migratorios}

En esta categoría se han establecido las siguientes subcategorías: a) ley y orden para gestionar la inmigración; b) conveniencia del consenso para regular la inmigración; c) necesidad de regulación a largo plazo y d) apelación al miedo o al alarmismo.

La primera subcategoría de este apartado se refiere a que el fenómeno de la inmigración debe tener como punto de referencia la ley y el orden. Esta idea es la preferida del Partido Popular (PP), si bien el Partido Socialista Obrero Español (PSOE) también la utiliza con frecuencia, sobre todo a partir de 2008. La siguiente cita del diario $A B C$ ilustra muy claramente la posición del PP, desde este planteamiento:

"La inmigración — destacó el vicepresidente [del Gobierno, del PP] - no puede ser infinita; ha de ser legal y ordenada" (...). El ministro Ángel Acebes sostuvo, por su parte, que España debe seguir siendo un país de acogida de inmigración, "pero 
legal". Recalcó que solo la ley garantiza la convivencia en paz "mientras que la ilegalidad o alegalidad solo genera conflictos" (Prieto, 2003: 12).

Otro de los discursos recurrentes es el que pone en relación el endurecimiento de la ley, con la excusa de impedir o dificultar la inmigración ilegal que está en manos de las mafias y que fomenta la trata de seres humanos, como refleja El Mundo:

José María Aznar cerrará su intensa campaña electoral aprobando el viernes en el Consejo de Ministros un endurecimiento de la ley de extranjería. (...) está basada en facilitar la situación de los inmigrantes legales, mientras se penaliza y se endurece la legislación contra los ilegales. (...) "Transigir con la ilegalidad supone renunciar al Estado de Derecho, que se basa en el cumplimiento de la ley y supone condenar al inmigrante a ser objeto de mafias y a la marginación social" (Garea, 2003: 7).

Otra subcategoría temática recurrente es la conveniencia de lograr un consenso lo más amplio posible a la hora de regular la inmigración. Dicho consenso se puede circunscribir al ámbito de los partidos políticos con representación parlamentaria en el Congreso de los Diputados o se puede ampliar a otros actores sociales como sindicatos, asociaciones de empresarios, ONG e inmigrantes. Así, el diario El País a través de un editorial en 2003: "El Gobierno debería imitar a los de otros países europeos con fuerte presión migratoria: tender la mano al conjunto de fuerzas políticas y sociales en aras de un consenso que afronte la inmigración como política de Estado y no como instrumento de lucha partidaria y reclamo electoral" (El País, 2003). El PSOE se muestra más activo en la búsqueda efectiva de un consenso político y social cuando toma la iniciativa en la elaboración del reglamento de extranjería del año 2004 (El País): "Rumí (...) por el Gobierno socialista: 'Hemos dado el primer paso y ha sido la elaboración del reglamento de la ley de extranjería, cuyo primer objetivo era llegar a un consenso, al diálogo y alejar la confrontación de este tema"' (Agencias, 2005).

Legislar a largo plazo es la tercera idea en esta categoría estatal, ya que la regulación de la inmigración carece de estabilidad, como demuestran los múltiples cambios legislativos desde los años 2000 hasta 2009, especialmente. Los partidos políticos tienden a ser muy cortoplacistas en su visión de esta materia y suelen solicitar un cambio inmediato en la ley de extranjería, como si eso solo bastara para solucionar el problema: "Las avalanchas en las fronteras de Ceuta y Melilla han puesto de relieve notables deficiencias en la legislación española sobre extranjería e inmigración" ( $A B C, 2005: 4)$.

En cuarto lugar, resulta significativa la presencia de una serie de referencias, cuyos autores son representantes políticos, que apelan al miedo o al alarmismo para justificar que hay que regular de forma restrictiva la inmigración. Se trata de apelaciones al "aquí no cabemos todos" o al "no hay recursos para todos". Así, El Mundo recoge en 2003:

Aznar afirma que España "estallará” si siguen llegando inmigrantes ilegales. (...): "Si sigue aumentando el número de inmigrantes a una velocidad vertiginosa, España acabará estallando". Hasta ahora, el jefe del Ejecutivo se había limitado a argumentar que la política del Gobierno consistía en defender la inmigración legal y 
en penalizar la ilegal. (...) Aznar subió el tono y pasó de decir que "España tiene una capacidad limitada de acogida" a realizar una aseveración claramente alarmista. (...) Aznar trató de convencer de la urgente necesidad de reformar la ley de extranjería. "Lo que está pasando con la inmigración en España es tan rápido y a tal velocidad que tenemos que adaptarnos rápidamente" (Del Barrio, 2003: 10).

La segunda forma de alarmismo tiene que ver con la escasez de recursos sociales que puede conllevar el aumento de la inmigración. Así, $A B C$, en un editorial del año 2004:

Los poderes públicos, especialmente Autonomías y Ayuntamientos, están asumiendo las prestaciones sociales de los inmigrantes, pero con un resultado preocupante en aquellas Comunidades con un alto índice de inmigración, donde las clases "nacionales" con rentas más bajas empiezan a sentir la competencia de los inmigrantes, cuyos parámetros socioeconómicos les hacen acreedores principales de las ayudas sociales $(A B C, 2004: 21)$.

Otro miedo tiene que ver con los problemas de convivencia que pueden generarse como consecuencia del descontrol de la inmigración irregular. Así, José Blanco, responsable del PSOE en 2010 dice $(A B C)$ : "Blanco aseguró que 'la mayor incongruencia es tener descontrolados a los irregulares en España para que se generen guetos absolutamente ingobernables que deterioren la convivencia"" (Morcillo, 2010: 20).

\subsubsection{La ley de extranjería en las comunidades autónomas (CC. AA.)}

Los diarios analizados presentan cuatro subcategorías: a) mayores competencias en inmigración para las CC. AA.; b) las particularidades de cada comunidad autónoma; c) los traslados de inmigrantes desde la Comunidad Canaria y d) la integración social de los inmigrantes.

En primer lugar, se produce el debate sobre la obtención de mayores competencias por parte de las CC.AA. en materia de acogida a los migrantes que se encuentran en sus respectivos territorios. Al final, las CC. AA. consiguen, tras la reforma legal de 2009, tener ciertas competencias sobre inmigración que antes eran privativas del Estado: El País: "A cambio de su apoyo, los nacionalistas catalanes han logrado ampliar las competencias de las comunidades autónomas, especialmente las de la Generalitat de Cataluña” (Bárbulo, 2009).

No obstante, como en otros ámbitos, si las competencias no van acompañadas de recursos económicos para desarrollarlas y gestionarlas, pueden ser más una carga que un logro. De ahí que se produzcan discusiones entre los representantes de las comunidades y del Gobierno central. El diario La Vanguardia muestra esta exigencia:

Las autonomías no podrán tener política de inmigración. El PP y el PSOE rechazarán hoy dar mayor participación a las comunidades autónomas en la política de inmigración durante el debate de la reforma de la ley de Extranjería en el Congreso. CiU votará en contra de la modificación. (...) alegó que son los gobiernos 
autonómicos los que al final deben asumir la prestación de servicios a las personas inmigradas (EFE, 2003: 32).

La segunda categoría temática se refiere al posicionamiento de cada comunidad autónoma según las circunstancias y el color político del gobierno autonómico respectivo. En Cataluña la Generalitat será más o menos beligerante con el Gobierno central dependiendo de si quien gobierna la comunidad sea CiU o el PSC (aunque sea en coalición). CiU, no obstante, al tener una posición bisagra en el Congreso de los Diputados, va a mantener, en relación con este asunto, un papel muy relevante e influyente, al carecer el Gobierno de Zapatero de mayoría absoluta para poder legislar sin apoyos. Por su parte, la Comunidad de Madrid, en manos del PP, se muestra contraria a cualquier cesión de competencias desde el Estado, y será beligerante en la reclamación de recursos para prestar servicios sociales a los inmigrantes, al ser una de las zonas con mayor densidad de población extranjera. Canarias, también, al ser un punto de entrada muy importante de extranjeros, por vía marítima, hace oír su voz para, por un lado, pedir al Gobierno que ponga freno a estas entradas y, por otro lado, para que descongestione su territorio de personas detenidas en sus centros de internamiento, enviándolas con destino a la península. El presidente (socialista) del Gobierno de Aragón en 2004, Marcelino Iglesias (M.I.), también se posiciona a favor de un mayor papel de las CC. AA. y de los ayuntamientos en materia de inmigración (Heraldo de Aragón): “¿Hay que cambiar la ley de extranjería? M.I.: 'Uno de los asuntos urgentes que vamos a tratar con el nuevo Gobierno central es la política de inmigración. Soy partidario de que las Comunidades Autónomas y los Ayuntamientos participemos y nos impliquemos de forma directa en todas las políticas relacionadas con la inmigración"” (Valero, 2004: 22).

El tercer tema relevante es, precisamente, el efecto que tiene en las CC. AA. el traslado de inmigrantes desde Canarias a la península, cuestión que genera tensiones entre el Gobierno y las CC. AA., tanto por el hecho de los traslados en sí, que se hacen de forma opaca, como por la proporción del reparto, que las comunidades más afectadas consideran injusto. Tanto el Gobierno central del PP como el del PSOE han utilizado este mecanismo para descongestionar los centros de internamiento de las islas, por lo que no parece haber grandes diferencias entre los dos grandes partidos en este tema. Así lo muestra $A B C$ : "El Gobierno fleta dos vuelos diarios desde Canarias a la Península con ilegales que abandona a su suerte. Solo en 2004 viajaron a Madrid 2.200 subsaharianos, que quedaron libres. El PSOE, que criticó la medida en la oposición, no solo sigue los pasos del PP sino que la intensifica" (Morcillo, 2005: 16).

Finalmente, en cuarto lugar, es recurrente la cuestión de cómo conseguir la integración de las personas migrantes. Aquí las CC.AA. tienen una labor básica y fundamental, que los medios reflejan con intensidad, especialmente en los diarios de ámbito regional como La Vanguardia, Heraldo de Aragón y El Periódico de Aragón. Este enfoque es especialmente frecuente a partir de la reforma de la ley de extranjería del año 2009, ya que, desde entonces, serán las CC. AA. quienes deban acreditar que los inmigrantes que deseen obtener determinados permisos han realizado el esfuerzo de integración requerido: "Integración. Las comunidades autónomas acreditarán el esfuerzo de integración de los inmigrantes como un 
complemento para suplir la carencia de otros requisitos a la hora de renovar sus documentos de residencia” (Bárbulo, 2009). En Cataluña se primará en 2010 el conocimiento del idioma catalán por parte de los inmigrantes, ya que facilitará que obtengan el certificado de arraigo que deben elaborar las CC.AA., lo que provoca bastante revuelo, sobre todo en medios nacionales. Así, en El Mundo:

Saber catalán será de gran ayuda para el inmigrante que quiera acceder a la nacionalidad por residencia. Al menos, a los que vivan en Cataluña. El Parlament aprobó ayer la Ley de Acogida, que premia a los recién llegados, si acreditan que conocen esa lengua, con beneficios para obtener papeles. (...) la consejera Capdevila cree que "las competencias lingüísticas son importantes para la integración", y afirmó que la lengua común es el catalán (Sastre, 2010: 18).

En $A B C$ se comenta también, y destaca: "El diputado del PP Rafael López criticó que "el catalán sea el peaje para que los inmigrantes obtengan papeles"" (Cañizares, 2010: 20).

\subsubsection{La ley de extranjería y los ayuntamientos}

Los temas que se tratan en esta subcategoría son: a) el escaso papel de los ayuntamientos en cuestiones de extranjería; b) la utilización de las cuestiones locales por los partidos políticos para llevarlas al debate de ámbito nacional en este tema y c) el uso del padrón municipal como herramienta para el control de la inmigración irregular.

Así, en primer lugar, la prensa analizada apenas articula sus mensajes alrededor del papel de los ayuntamientos en cuestiones jurídicas relacionados con la ley de extranjería; algo lógico, ya que las entidades locales no tienen competencias jurídicas en la materia. Solo cuando algunos ayuntamientos se exceden en sus competencias, como en el caso de la prohibición del burka en Lérida, la prensa se hace eco. Así, en El Mundo:

El Ayuntamiento de Lérida será el primero en prohibir el burka, aunque no lo hará en la vía pública, como se había propuesto en un principio, porque no tiene potestad jurídica. El veto se limitará a los edificios municipales (...). Esta iniciativa ha abierto el debate en Cataluña, donde algunos sectores han puesto en duda su idoneidad, ya que apuestan antes por hacer pedagogía y difundir la igualdad entre hombres y mujeres, y cuestionan que sea realmente necesaria aduciendo la poca presencia de velos integrales en las calles (Serret, 2010, p.16).

También cuando se produce la negativa a empadronar a personas inmigrantes en situación irregular por parte de los municipios de Vic (Barcelona) y Alcorcón (Madrid). Por ejemplo en El Mundo:

El alcalde de Vic (Barcelona), Josep Maria Vila d'Abadal (CiU), se resignó ayer a acatar la ley y a inscribir a todos los inmigrantes, también a los que están a la espera de obtener el permiso de residencia. Advirtió de que la decisión de empadronar a inmigrantes en situación irregular "puede tener grandes costes sociales en el futuro" 
si no se discuten los criterios para llevar a cabo la inscripción en el padrón (Ribalaygue, 2010: 5).

Para el caso de Torrejón de Ardoz (Madrid), El Mundo plasma la contradicción:

Los grupos parlamentarios del PSOE e IU en la Asamblea de Madrid señalaron ayer a Torrejón de Ardoz para pedirle a la presidenta de la Comunidad madrileña, Esperanza Aguirre, que se cumpla la ley también en el citado municipio en lo referente al empadronamiento. (...) Pedro Rollán (PP): "Es más, en Vic no empadronan a sin papeles; en Torrejón sí. Lo que pasa es que hay unos requisitos de habitabilidad mínimos para evitar pisos patera y camas calientes y que esta gente sufra en manos de unos desalmados" (Cruz, 2010: 5).

Circunstancialmente, los ayuntamientos aparecen en los medios analizados quejándose de la falta de recursos para la prestación de servicios sociales que deben dar a las personas extranjeras en situación irregular, o bien para favorecer la mejor integración social, cuestiones ambas en las que coinciden con las CC. AA. Los municipios también quieren tener una mayor participación en la toma de las decisiones en este ámbito. Así lo refleja La Vanguardia:

Los ayuntamientos de Tortosa y El Vendrell se sumarán a los municipios que demandarán que el reglamento de la ley de Extranjería permita a los municipios tener una mayor influencia en la decisión del Gobierno sobre la permanencia de extranjeros en nuestro país (Margalef, 2010:5).

En segundo término, los partidos políticos no pierden ocasión para convertir cuestiones locales en generales, llevándolas al debate nacional, algo a lo que contribuye la prensa. Por ejemplo, El Mundo: "El PP considera necesario que el ministro de Trabajo e Inmigración, Celestino Corbacho, acuda al Congreso a explicar lo que está ocurriendo en el Ayuntamiento de Vic (Barcelona), que se niega a empadronar a los inmigrantes que no tienen documentación a pesar de que las corporaciones están obligadas legalmente a inscribir en el padrón municipal a todas las personas que viven en su municipio" (Yanel, 2010:17).

Por último, el tema con mayor presencia en este ámbito local es, sin duda, el relacionado con el uso del padrón municipal de habitantes como herramienta de control de la inmigración irregular, sobre todo mediante la posibilidad legal que se abre en 2003 para que la Policía pueda cruzar los datos de diversas administraciones públicas con los datos del padrón. Esto provoca una agria polémica entre los defensores de los derechos humanos y el partido del Gobierno en aquel momento, que es el PP, si bien con acuerdo del PSOE, como se ve en $A B C$ :

Maragall discrepa del PSOE y pide que no se cedan datos de inmigrantes a la Policía. Los socialistas pactaron con el PP esta medida incluida en la reforma de la Ley de Extranjería. (...) El PSOE, que acaba de pactar con el PP la reforma de la Ley de Extranjería, está de acuerdo en que la Policía pueda hacer uso de los datos 
del padrón como fuente de información para el control de la inmigración (Cañizares, 2003: 21).

\subsection{Las fuentes informativas principales empleadas por la prensa}

En este epígrafe se presentan los resultados del análisis de contenido cuantitativo realizado, que han permitido determinar: a) cuáles son las fuentes principales empleadas por cada uno de los medios y en el conjunto de todos ellos cuando han tratado el tema objeto de estudio, y b) la prevalencia de las fuentes oficiales o las de la sociedad civil.

Tabla 2. Las fuentes principales empleadas por la prensa (2003-2016)

\begin{tabular}{|c|c|c|c|c|c|c|c|c|}
\hline & $\begin{array}{l}\text { El } \\
\text { País }\end{array}$ & $\begin{array}{l}\text { El } \\
\text { Mundo }\end{array}$ & $\mathrm{ABC}$ & $\begin{array}{l}\text { Heraldo } \\
\text { de } \\
\text { Aragón }\end{array}$ & $\begin{array}{l}\text { El } \\
\text { Periódico } \\
\text { de } \\
\text { Aragón }\end{array}$ & $\begin{array}{l}\text { La } \\
\text { Vanguardia }\end{array}$ & \multicolumn{2}{|c|}{$\begin{array}{c}\text { Conjunto } \\
\text { de } \\
\text { diarios }\end{array}$} \\
\hline & \multicolumn{6}{|c|}{$\%$ dentro del diario } & $\begin{array}{c}\mathrm{N}^{\circ} \text { de } \\
\text { unidades }\end{array}$ & $\%$ \\
\hline Gobierno o Adm. central & 27,3 & 26,2 & 25,6 & 15,7 & 18,2 & 26,1 & 50 & 22,6 \\
\hline $\begin{array}{l}\text { Miembro de partido político } \\
\text { de la oposición }\end{array}$ & 21,2 & 21,4 & 17,9 & 3,9 & 18,2 & 26,1 & 37 & 16,7 \\
\hline $\begin{array}{l}\text { Gobierno o Adm. } \\
\text { autonómica }\end{array}$ & 3,0 & 16,7 & 23,1 & 13,7 & 3,0 & 13,0 & 28 & 12,7 \\
\hline ONG & 6,1 & 7,1 & 0 & 11,8 & 15,2 & 8,7 & 18 & 8,1 \\
\hline Sindicato & 0 & 0 & 0 & 13,7 & 21,2 & 0 & 14 & 6,3 \\
\hline El propio medio (editorial) & 18,2 & 2,4 & 5,1 & 5,9 & 6,1 & 0 & 14 & 6,3 \\
\hline $\begin{array}{l}\text { El propio medio } \\
\text { (periodista o columnista) }\end{array}$ & 3,0 & 4,8 & 2,6 & 9,8 & 0 & 21,7 & 14 & 6,3 \\
\hline Abogado & 0 & 0 & 2,6 & 9,8 & 0 & 0 & 6 & 2,7 \\
\hline Experto & 6,1 & 2,4 & 5,1 & 2,0 & 0 & 0 & 6 & 2,7 \\
\hline Testimonio de inmigrante & 0,0 & 4,8 & 2,6 & 2,0 & 3,0 & 0 & 5 & 2,3 \\
\hline Ayuntamiento & 3,0 & 4,8 & 0 & 0 & 0 & 4,3 & 4 & 1,8 \\
\hline Cuerpos de seguridad & 0 & 2,4 & 5,1 & 0 & 3,0 & 0 & 4 & 1,8 \\
\hline Asociación de inmigrantes & 6,1 & 0 & 0 & 0 & 6,1 & 0 & 4 & 1,8 \\
\hline Defensor del Pueblo & 0 & 2,4 & 2,6 & 3,9 & 0 & 0 & 4 & 1,8 \\
\hline Tribunales de Justicia & 3,0 & 2,4 & 5,1 & 0 & 0 & 0 & 4 & 1,8 \\
\hline Otros & 0 & 0 & 2,6 & 3,9 & 3,0 & 0 & 4 & 1,8 \\
\hline $\begin{array}{l}\text { No consta desconocida } \\
\text { o anónima }\end{array}$ & 0 & 2,4 & 0 & 3,9 & 3,0 & 0 & 4 & 1,8 \\
\hline Iglesia católica & 3,0 & 0 & 0 & 0 & 0 & 0 & 1 & 0,5 \\
\hline Total (\% y n ${ }^{\circ}$ de uds.) & $\begin{array}{c}100 \% \\
(33)\end{array}$ & $\begin{array}{c}100 \% \\
(42)\end{array}$ & $\begin{array}{l}100 \% \\
(39)\end{array}$ & $\begin{array}{l}100 \% \\
(51)\end{array}$ & $\begin{array}{c}100 \% \\
(33)\end{array}$ & $\begin{array}{c}100 \% \\
(23)\end{array}$ & 221 & $100 \%$ \\
\hline
\end{tabular}

Fuente: elaboración propia. 
Como ya se adelantó en el epígrafe dedicado a fuentes y metodología, son 18 las fuentes principales empleadas, tal como se puede observar en la Tabla 2.

En el conjunto de los diarios, la fuente principal más frecuente es la Administración o Gobierno central (22,6\%), seguida de los miembros de partidos políticos de la oposición (16,7\%) y los Gobiernos o Administraciones autonómicas $(12,7 \%)$. Las ONG son la cuarta fuente $(8,1 \%)$ en este ranking y la primera de la sociedad civil.

Esta variedad de fuentes la sintetizamos en tres categorías en función de su origen: oficiales, de la sociedad civil y los propios medios de comunicación (ver Tabla 3) a partir de los criterios establecidos en el apartado de "Fuentes y Metodología". Lo primero que llama la atención es que las fuentes oficiales suponen, en el conjunto de los diarios, un 59,3\% del total, mientras que las de la sociedad civil ocupan solo el 26,2\%. Los medios (a través de sus editoriales y columnistas, especialmente) son fuente principal el $12,7 \%$ de las veces. En definitiva, las fuentes oficiales duplican a las de la sociedad civil. Por diarios, es reseñable la preponderancia de las fuentes oficiales en la prensa nacional y en $L a$ Vanguardia. Son los diarios regionales aragoneses los que ofrecen un enfoque diferente, al dotar de un mayor protagonismo a las fuentes de la sociedad civil en relación con el resto de medios.

Tabla 3. Fuentes principales: fuentes oficiales frente a fuentes de la sociedad civil

\begin{tabular}{|l|c|c|c|c|c|c|c|c|}
\hline & El País & $\begin{array}{l}\text { El } \\
\text { Mundo }\end{array}$ & ABC & $\begin{array}{l}\text { Heraldo } \\
\text { de Aragón }\end{array}$ & $\begin{array}{l}\text { El Periódico } \\
\text { de Aragón }\end{array}$ & $\begin{array}{l}\text { La } \\
\text { Vanguardia }\end{array}$ & \multicolumn{2}{c|}{$\begin{array}{c}\text { Conjunto de } \\
\text { diarios } \\
\left(\mathrm{n}^{\mathrm{o}} \text { de uds. y } \%\right)\end{array}$} \\
\hline $\begin{array}{l}\text { Fuentes } \\
\text { oficiales }\end{array}$ & $57,6 \%$ & $76,2 \%$ & $79,5 \%$ & $37,3 \%$ & $42,4 \%$ & $69,6 \%$ & 131 & $59,3 \%$ \\
\hline Sociedad civil & $21,2 \%$ & $14,3 \%$ & $12,8 \%$ & $43,1 \%$ & $48,5 \%$ & $8,7 \%$ & 58 & $26,2 \%$ \\
\hline El propio medio & $21,2 \%$ & $7,1 \%$ & $7,7 \%$ & $15,7 \%$ & $6,1 \%$ & $21,7 \%$ & 28 & $12,7 \%$ \\
\hline No consta & $0 \%$ & $2,4 \%$ & $0 \%$ & $3,9 \%$ & $3,0 \%$ & $0 \%$ & 4 & $1,8 \%$ \\
\hline Total & $100 \%$ & $100 \%$ & $100 \%$ & $100 \%$ & $100 \%$ & $100 \%$ & 221 & $100 \%$ \\
\hline
\end{tabular}

Fuente: elaboración propia a partir de los datos de la Tabla 2.

\subsection{Los discursos y las retóricas discursivas en torno a la inmigración}

En este apartado se analiza qué tipología de discursos (proactivos, reactivos o mixtos), según el modelo de Zapata-Barrero (2009) ya explicado, se encuentra en los diferentes diarios investigados (ver Tabla 4). Como ya se ha explicado, son cuatro las retóricas que se pueden encontrar en los discursos que tienen que ver con la multiculturalidad y la inmigración: dos de tipo reactivo (tradicionalismo y ciudadanismo) y dos de tipo proactivo (igualdad y ciudadanía).

$\mathrm{Al}$ observar estos datos, lo primero que destaca es que, en el conjunto de los diarios, la retórica con más peso es la proactiva de igualdad $(32,1 \%)$, seguida, ya a 
mucha distancia, de las dos retóricas reactivas de tradicionalismo $(14,5 \%)$ y ciudadanismo (14\%). Solo en el $8,6 \%$ de las unidades no ha sido posible encontrar una retórica siguiendo este modelo.

Tabla 4. Retóricas reactivas y proactivas presentes en los diarios analizados (2003-2016)

\begin{tabular}{|c|c|c|c|c|c|c|c|c|}
\hline & El País & El Mundo & $\mathrm{ABC}$ & $\begin{array}{l}\text { Heraldo } \\
\text { de }\end{array}$ & $\begin{array}{l}\text { El } \\
\text { Periódico } \\
\text { de }\end{array}$ & $\begin{array}{l}\text { La } \\
\text { Vanguardia }\end{array}$ & \multicolumn{2}{|c|}{$\begin{array}{l}\text { Conjunto de } \\
\text { diarios }\end{array}$} \\
\hline & \multicolumn{6}{|c|}{$\%$ dentro del diario } & $\begin{array}{l}\text { N. } \\
\text { de }\end{array}$ & $\%$ \\
\hline $\begin{array}{l}\text { Tradicionalismo } \\
\text { (reac.) }\end{array}$ & 6,1 & 16,7 & 35,9 & 7,8 & 6,1 & 13,0 & 32 & 14,5 \\
\hline $\begin{array}{l}\text { Ciudadanismo } \\
\text { (react.) }\end{array}$ & 21,2 & 23,8 & 7,7 & 3,9 & 6,1 & 30,4 & 31 & 14,0 \\
\hline $\begin{array}{l}\text { Igualdad } \\
\text { (proact.) }\end{array}$ & 24,2 & 23,8 & 5,1 & 56,9 & 54,5 & 17,4 & 71 & 32,1 \\
\hline $\begin{array}{l}\text { Ciudadanía } \\
\text { (proact.) }\end{array}$ & 12,1 & 2,4 & 2,6 & 3,9 & 9,1 & 0 & 11 & 5,0 \\
\hline $\begin{array}{l}\text { Tradicionalismo y } \\
\text { ciudadanismo } \\
\text { (react.) }\end{array}$ & 9,1 & 7,1 & 12,8 & 2,0 & 0 & 8,7 & 14 & 6,3 \\
\hline $\begin{array}{l}\text { Igualdad y } \\
\text { ciudadanía } \\
\text { (proact.) }\end{array}$ & 6,1 & 2,4 & 0 & 3,9 & 6,1 & 4,3 & 8 & 3,6 \\
\hline $\begin{array}{l}\text { Tradicionalismo e } \\
\text { igualdad (mixta) }\end{array}$ & 0 & 2,4 & 15,4 & 5,9 & 0 & 4,3 & 11 & 5,0 \\
\hline $\begin{array}{l}\text { Tradicionalismo y } \\
\text { ciudadanía (mixta) }\end{array}$ & 0 & 2,4 & 0 & 0 & 0 & 0 & 1 & 0,5 \\
\hline $\begin{array}{l}\text { Ciudadanismo e } \\
\text { igualdad (mixta) }\end{array}$ & 12,1 & 11,9 & 10,3 & 9,8 & 0 & 13,0 & 21 & 9,5 \\
\hline $\begin{array}{l}\text { Ciudadanismo y } \\
\text { ciudadanía (mixta) }\end{array}$ & 3,0 & 0 & 0 & 0 & 3,0 & 0 & 2 & 0,9 \\
\hline $\begin{array}{l}\text { Ninguno o no se } \\
\text { identifica }\end{array}$ & 6,1 & 7,1 & 10,3 & 5,9 & 15,2 & 8,7 & 19 & 8,6 \\
\hline $\begin{array}{l}\text { Total } \\
\text { (\% y n de uds.) }\end{array}$ & $\begin{array}{c}100 \% \\
(33)\end{array}$ & $\begin{array}{l}100 \% \\
(42)\end{array}$ & $\begin{array}{l}100 \% \\
(39)\end{array}$ & $\begin{array}{l}100 \% \\
(51)\end{array}$ & $\begin{array}{c}100 \% \\
(33)\end{array}$ & $\begin{array}{c}100 \% \\
(23)\end{array}$ & 221 & $100 \%$ \\
\hline
\end{tabular}

Fuente: elaboración propia.

Las retóricas pueden encontrarse de forma única en las unidades de análisis o combinadas entre sí, lo que generará discursos proactivos si incorporan una o dos de las retóricas de este tipo en una misma unidad; reactivos si se trata de retóricas de esta modalidad, o mixtos si la combinación incluye una retórica proactiva y otra reactiva (Gráfico 1). 
Gráfico 1. Tipología de discursos presente en los diarios analizados (2003-2016)

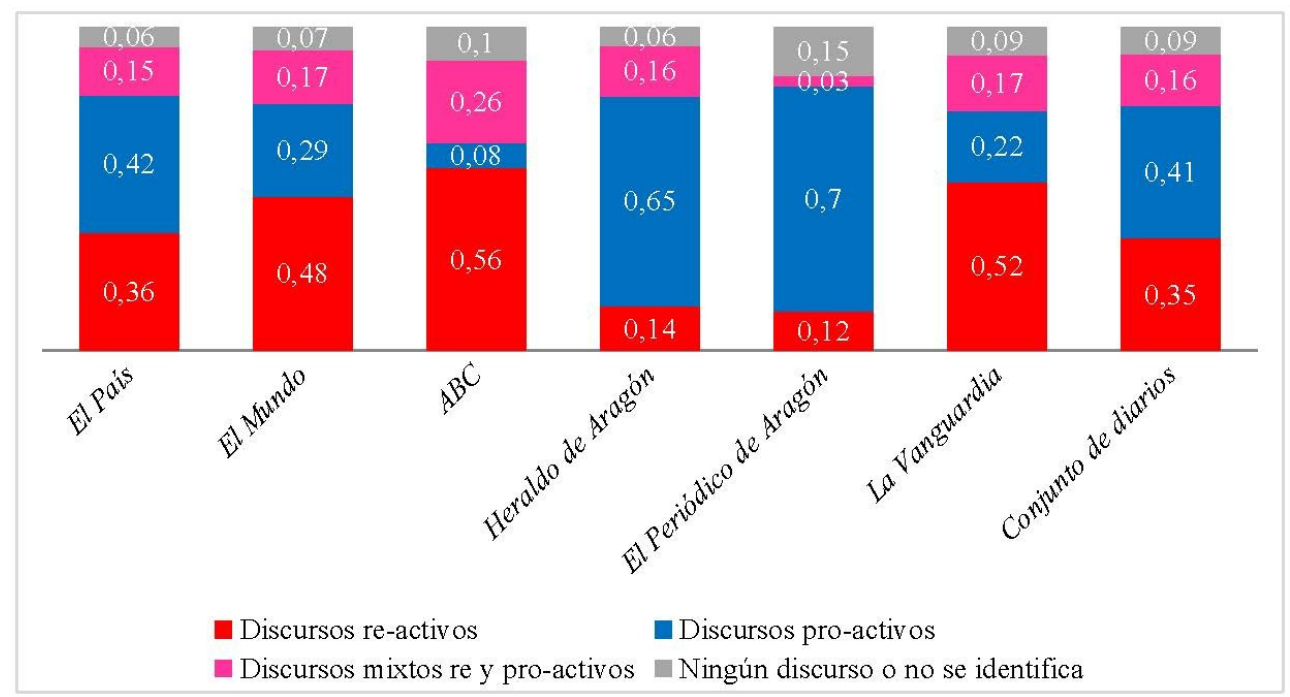

Fuente: elaboración propia a partir de los datos de la Tabla 4.

Así, cuando los diarios tratan el tema de las políticas públicas y la ley de extranjería, el $40,7 \%$ de las unidades muestra un discurso proactivo, mientras un $34,8 \%$ son reactivos y un $15,8 \%$, mixto entre ambos. Por diarios, El País obtiene resultados muy cercanos al del conjunto de los medios, mientras que El Mundo y La Vanguardia obtienen resultados similares: dan mayor peso a los discursos reactivos $(47,6 \%$ el primero y $52,2 \%$ el segundo) que a los proactivos $(28,6 \% \mathrm{El}$ Mundo y $21,7 \%$ La Vanguardia). Un caso aparte es el de $A B C$, donde predominan los discursos reactivos $(56,4 \%)$ sobre los escasísimos proactivos $(7,7 \%)$. Por el contrario, los diarios aragoneses tienen un enfoque totalmente contrario, ya que sus discursos son muy proactivos (64,7\% en Heraldo de Aragón y $69,7 \%$ en El Periódico de Aragón). Los discursos mixtos se encuentran con más frecuencia en $A B C(25,6 \%)$.

\section{Conclusiones}

A la hora de sacar conclusiones globales a partir de las preguntas de investigación planteadas, en primer lugar, observamos una vinculación entre la agenda mediática y la tipología de las fuentes informativas. Así, los temas relevantes para la prensa en relación con las políticas públicas y la ley de extranjería están muy vinculados con las propuestas de las fuentes oficiales, que se ha visto que son mayoritarias, especialmente las del Gobierno de la nación y las de los partidos políticos de la oposición, como contrapunto. El interés por la ley y el orden, el consenso y la regulación a largo plazo, los intereses de los Gobiernos autonómicos o el papel de los ayuntamientos son ejemplos de ello. 
En segundo lugar, se aprecia la relación existente entre la selección de las fuentes y los discursos. Como hemos visto, las fuentes oficiales prevalecen en el conjunto de los diarios analizados, con la excepción de los dos diarios aragoneses. Estos diarios regionales prefieren las fuentes de la sociedad civil, por lo que su discurso es más proactivo, como se ha comprobado. Así, cuando el sesgo es excesivo hacia las fuentes oficiales respecto a las de la sociedad civil, acaban predominando los discursos reactivos. Es lo que le ocurre a La Vanguardia, donde la diferencia de 60,9 puntos porcentuales a favor de las fuentes oficiales se traduce en una discrepancia de 30,5 puntos a favor de los discursos reactivos frente a los proactivos. En el caso de El Mundo, la diferencia de 61,9 puntos entre fuentes oficiales y de la sociedad civil solo se convierte en una distancia de 19 puntos entre discursos reactivos y proactivos, mientras que en $A B C$, la discrepancia de 66,7 puntos entre fuentes oficiales y de la sociedad civil hace que los discursos reactivos superen a los proactivos en 48,7 puntos porcentuales.

Queda así plenamente demostrada la relación directa, ya apuntada por los autores citados, entre la selección de fuentes y el resultado discursivo. Y es que, además, en relación con el tratamiento de la inmigración, se echa en falta una mayor presencia de los principales protagonistas de las políticas de extranjería: los propios inmigrantes. Los medios nacionales y La Vanguardia (que se asemeja a ellos) abusan, desde nuestra perspectiva, de las fuentes oficiales, a las que tienen fácil acceso. Los diarios aragoneses, en cambio, por su diferente filosofía editorial y recursos, prefieren aproximarse al tema desde una perspectiva más cercana y esto queda perfectamente reflejado en los discursos presentados en el Gráfico 1.

Como reflexión final, los resultados parecen indicar que los medios analizados (salvo los estrictamente regionales) no cumplen con su función de estimuladores y orientadores de las opiniones y deseos de los ciudadanos, dentro de la esfera pública. En concreto, no compelen al sistema político a adaptarse y ser más transparente, sino que los medios aparecen más como meros transmisores de los argumentos de las fuentes oficiales que como auténticos mediadores sociales que promuevan el diálogo entre la ciudadanía (la sociedad civil, incluidos los inmigrantes) y los representantes de los poderes públicos.

\section{Bibliografía}

$A B C$ (2004): "Política de inmigración: integrar para ganar", en $A B C, 2$ de marzo, p. 21. $A B C$ (2005): "Defectos en las normas de extranjería", en $A B C, 9$ de octubre, p. 4.

Agencias (2005): "Presentadas 237.067 solicitudes de inmigrantes al proceso de regulación en siete semanas", en El País, 29 de marzo. Disponible en:

http://elpais.com/elpais/2005/03/29/actualidad/1112084222_850215.html

[Consulta: 20 de septiembre de 2018]

Bañón, A. y J. Fornieles (2008): Manual sobre comunicación e inmigración, San Sebastián, Tercera Prensa.

Bárbulo, T. (2009): “El Gobierno reforma la Ley de Extranjería con los nacionalistas”, en El País, 30 de octubre. Disponible en:

www.elpais.com/diario/2009/10/30/espana/1256857210_850215.html

[Consulta: 5 de mayo de 2017] 
Berelson, B. (1952): Content Analysis in Communication Research, Nueva York, Free Press.

Berganza Conde, M. y M. Á. Chaparro Domínguez (2012): "El rigor en la prensa: principales características y diferencias en el uso de las fuentes en los periódicos gratuitos y de pago", Zer: Revista de Estudios de Comunicación, 17(32), pp. 29-49.

Borrat, H. (1989): El periódico, actor del sistema político, Barcelona, Gustavo Gili.

Cañizares, M. J. (10 de octubre de 2003): "Maragall discrepa del PSOE y pide que no se cedan datos de inmigrantes a la Policía", en $A B C$, p. 21

Cañizares, M. J. (29 de abril de 2010): "La Generalitat relega el castellano para dar el carné de arraigo a los inmigrantes", en $A B C$, p. 20.

Casero, A. (2007): "Discurso mediático, inmigración e ilegalidad: legitimar la exclusión a través de la noticia”, en Zapata-Barrero, R., Discursos sobre la inmigración en España. Los medios de comunicación, los parlamentos y las administraciones, Barcelona, Fundación CIDOB, pp. 69-90.

Casero, A. y P. López (2012): "La evolución del uso de las fuentes informativas en el periodismo español", en III Congreso Internacional "Comunicación y Riesgo" . Tarragona, Universitat Rovira i Virgili.

Cortes Generales de España (2000): Ley orgánica 4/2000, de 11 de enero, sobre derechos y libertades de los extranjeros en España y su integración social. Disponible en: https://www.boe.es/buscar/act.php?id=BOE-A-2000-544 [Consulta: 6 de mayo de 2017]

Cruz, M. (16 de enero de 2010): "PSOE e IU señalan ahora a Torrejón de Ardoz", en El Mundo, p.5.

De Fontcuberta, M. (1993): La noticia: Pistas para percibir El Mundo, Barcelona, Paidós.

De la Fuente, M. (2007): “Argumentación e inmigración. Reflexiones sobre algunas estrategias empleadas en los medios de comunicación”, en Bañón, A. M., Discurso periodístico y procesos migratorios, San Sebastián, Tercera Prensa, pp. 343-374.

Del Barrio, A. (21 de mayo de 2003): “Aznar afirma que España 'estallará' si siguen llegando inmigrantes ilegales", en El Mundo, p. 10.

Delgado, L. (2007): "Argumentación gubernamental y política de inmigración", en Van-Dijk, T. A. y R. Zapata-Barrero, Discursos sobre la inmigración en España. Los medios de comunicación, los parlamentos y las administraciones, Barcelona, Fundación CIDOB, pp. 201-221.

El País (2003): "Reforma a peor” en El Pais, 22 de diciembre. Disponible en: http://elpais.com/diario/2003/12/22/opinion/1072047602_850215.html [Consulta: 17 de mayo de 2017]

Garea, F. (20 de mayo de 2003): “Aznar anuncia que endurecerá la ley contra la inmigración ilegal el último día de campaña", en El Mundo, p. 7.

Gomis, L. (1991): Teoría del periodismo. Cómo se forma el presente, Barcelona, Paidós.

Habermas, J. (1974): “La Esfera de lo Público", New German Critique(3), pp. 123-130.

Habermas, J. (2006): "Political Communication in Media Society: Does Democracy Still Enjoy an Epistemic Dimension? The Impact of Normative Theory on Empirical Research", Communication Theory(16), pp. 411-426.

Habermas, J. (2009): "Media, Markets and Consumers: The Quality Press as the Backbone of the Public Sphere", en J. Habermas, Europe The Faltering Project, Nueva York, Polity Press, pp. 131-137.

Krippendorff, K. (1990): Metodología de análisis de contenido, Barcelona, Paidós. 
López, M. (1995): Cómo se fabrican las noticias. Fuentes, selección y planificación, Barcelona, Paidós.

Margalef, O. (23 de diciembre de 2010): "El Vendrell y Tortosa debatirán la inmigración”, en La Vanguardia, p. 5.

Márquez, E. (2007): "La gestión parlamentaria del discurso político sobre inmigración en España”, en Zapata-Barrero, R., Discursos sobre la inmigración en España. Los medios de comunicación, los parlamentos y las administraciones, Barcelona, Fundación CIDOB, 93-127.

Martín, L. R. y R.Whittaker (1998): Poder-decir o el poder de los discursos, Madrid, Ed. U. Autónoma de Madrid.

Morcillo, C. (17 de enero de 2005): "El Gobierno fleta dos vuelos diarios desde Canarias a la Península con ilegales que abandona a su suerte", en $A B C$, p. 16.

Morcillo, C. (21 de enero de 2010): "Vic calla tras el dictamen de la Abogacía del Estado que avala empadronar "ilegales"”, en $A B C$, p. 20.

Ospina Hurtado, X. (2004): El papel de las fuentes oficiales en la calidad del periodismo colombiano, Bogotá, Centro de Competencia en Comunicación para América Latina-Proyecto Antonio Nariño. Disponible en:

www.c3fes.net

[Consulta: 10 de diciembre de 2016]

Pecourt Gracia, J. (2015): "La esfera pública digital y el activismo político", Política y Sociedad, 52 (1), pp. 75-98.

http://dx.doi.org/10.5209/rev_POSO.2015.v1.n52.45423

Peña, D. y J. Romo (1997): Introducción a la Estadística para las Ciencias Sociales, Madrid, McGraw-Hill Interamericana de España.

Pérez Curiel, C. (2005): Tesis doctoral: Estudio de las fuentes en marco del periodismo especializado: estrategias de selección y tratamiento de las fuentes en las secciones periodísticas de El Mundo y El País. Disponible en:

https://idus.us.es/xmlui/handle/11441/24400

[Consulta: 31 de octubre de 2017]

Prieto, M. (13 de enero de 2003): "El Gobierno impulsa más medidas para castigar con dureza a las mafias que trafican con inmigrantes", en $A B C$, p. 12.

Ribalaygue, J. (22 de enero de 2010): "Marcha atrás del alcalde de Vic tras el informe oficial", en El Mundo, p. 5.

Rodríguez Cruz, I. y M. J. Bezunartea Valencia (2015): “El medio ambiente, supeditado a la conveniencia de la economía y la política. Diagnóstico de la información a partir de sus fuentes", Zer: Revista de Estudios de Comunicación, 20(39), pp. 85-100. https://doi.org/10.1387/zer.15523

Sastre, D. G. (29 de abril de 2010): “Premio para los inmigrantes que aprendan catalán”, en El Mundo, p. 18.

Serret, M. (28 de mayo de 2010): "Lérida veta el burka en edificios públicos", en $E l$ Mundo, p. 16.

Thompson, J. B. (1996): "La teoría de la esfera pública”, Voces y culturas (10).

Valero, J. L. (11 de abril de 2004): "Marcelino Iglesias: 'Si el PP hubiera ganado en Aragón, yo habría tenido que convocar elecciones anticipadas", en Heraldo de Aragón, p. 22.

Van-Dijk, T. A. (2007): "El racismo y la prensa en España”, en Bañón, A. M., Discurso periodístico y procesos migratorios, San Sebastián, Tercera Prensa, pp. 27-80. 
Van-Dijk, T. A. (2008): "Reproducir el racismo: el rol de la prensa", en Checa, F., La inmigración sale a la calle: Comunicación y discursos políticos sobre el fenómeno migratorio, Barcelona, Icaria.

Van-Dijk, T. A. (2009): Discurso y poder, Barcelona, Gedisa.

Van-Dijk, T. A. y R. Zapata-Barrero (2007): "Introducción: inmigración y discurso", en Zapata-Barrero, R. y T. A. Van-Dijk, Discursos sobre la inmigración en España. Los medios de comunicación, los parlamentos y las administraciones, Barcelona, Fundación CIDOB, pp. 9-14.

Wodak, R. y R. Meyer (2003): Métodos de análisis crítico del discurso, Barcelona, Gedisa.

Yanel, A. (18 de enero de 2010): "El PP pide que Corbacho hable de Vic en el Congreso", en El Mundo, p. 17.

Zapata-Barrero, R. (2008): Multiculturalidad e inmigración, Madrid, Síntesis.

Zapata-Barrero, R. (2009): Fundamentos de los discursos políticos en torno a la inmigración, Madrid, Trotta.

Zapata-Barrero, R. y T. V. Van Dijk (2007): Discursos sobre la inmigración en España. Los medios de comunicación, los parlamentos y las administraciones, Barcelona, CIDOB. 